\title{
Analysis of Anomaly in the Behavior Characteristics of Operators in HSMM-based Multi-UAV Supervisory Control System
}

\author{
Hongbin Zhang ${ }^{1}$, Jian Yuan ${ }^{2}$, Liyong $\mathrm{Ma}^{3}$ \\ ${ }^{1}$ Department of Electronic Engineering, Tsinghua University, Beijing, 100084, China \\ ${ }^{2}$ Department of Electronic Engineering, Tsinghua University, Beijing, 100084, China \\ ${ }^{3}$ Communication Training Base of General Staff, Zhangjiakou, 075100,China
}

Keywords: HSMM, UAV, HSC, behavior characteristics.

\begin{abstract}
Solo operation of multi-unmanned Aerial Vehicle (UAV) will be the trend of future, and operators play a significant role in the UAV supervisory control system, but human may be impacted by all kinds of factors, which may decline the performance of UAV system. In this paper, modeling is conducted for the behavioral characteristics of operators in the multi-UAV supervisory control system with improved Hidden semi-Markov Model (HSMM), and the relationship between the predication result and anomaly of HSMM model is analyzed. This method can be applied for monitoring the anomaly in the behaviors of operators, which is of strong and practical significance in the multi-UAV supervisor control system and Human Supervisory Control (HSC) system.
\end{abstract}

\section{Introduction}

The current Unmanned Aerial Vehicle (UAV) system still adopts the manipulation of UAV by several operators. In order to realize the manipulation of multi-UAV by single operator, a lot of studies have been conducted in the automation of multi-UAV control and man-machine work allocation, etc. [1]; with the improvement of automation of UAV system, the operation of multiUAV by single operator will be favourable in the future, and it will also become a significant part in the future network centric warfare [2].

There are many differences in the control of UAV by single operator and current UAV control system. In the traditional UAV system, operators mainly control the UAV manually, while in multiUAV control system, the automatic system takes charges of the majority of operation, and the operators are mainly responsible for the supervision and control of UAV system, as well as the intervention and decision in emergencies beyond the handling of automatic system. Consequently, the future multi-UAV system is a typical Human Supervisory Control (HSC) system [3].

In this paper, modeling is conducted for the behaviors of operators in the UAV HSC system with Hidden semi-Markov Model (HSMM) with the experimental data as the drive. When there are anomalies in the behavioral characteristics of operators, the relationship between the prediction result and abnormal behavior of HSMM model is analyzed. With this method, the real-time supervision and analysis of the behavioral characteristic anomaly is implemented in the UAV supervision and control or other HSC scenes.

\section{Background}

There is no real multi-UAV supervisory control system of single operator. Massachusetts Institute of Technology (MIT) developed Research Environment for Supervisory Control of Heterogeneous Unmanned Vehicles (RESCHU) [4] and conducted a series of studies. Fig. 1 is the user interface of RESCHU which simulates the situation where an operator manipulates multiple UAVs to complete the reconnaissance mission under reconnaissance scene in the future network centric warfare. 


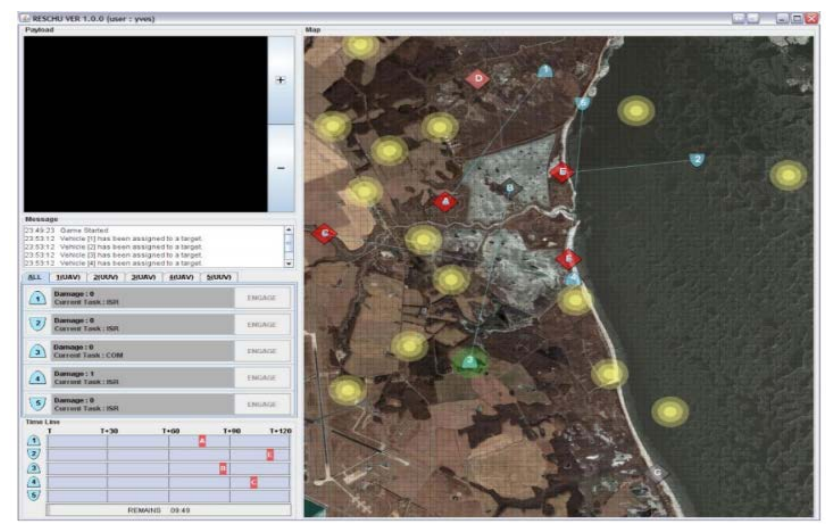

Figure 1: The RESCHU interface

On this platform, in order to accomplish the reconnaissance mission, the operator should plan the path of UAV at first, so as to minimize the running path of UAV. When UAV approaches the object, it will encounter a dangerous region and the operator should avoid this region in time. After UAV reaches the task object, the operator needs to search pictures of the object region transferred by UAV and confirm the object of reconnaissance mission.

It is pointed out in [5] and [6] that in practical environment, experienced operators tend to make a decision according to the previous cognition and meanwhile the operator of UAV must operate the UAV system according to the preset standard operating procedure; such behaviors might form a fixed mode easily. Therefore, under UAV HSC system, human operation behavior and system behavior have some rules. Behavioral data of the operator can be abstracted into a discrete time sequence and a suitable model can be adopted for modeling [7].

Under HSC scene of UAV, human behaviors will be disturbed by many factors. The future observed value is related to the past. Hidden semi-Markov Model (HSMM) is the extension of hidden Markov model (HMM) and it is obtained by adding state duration into HMM [8]. HSMM has overcome the strict geometric distribution restriction of HMM when describing state duration and the duration of any distribution can be depicted. In recent years, HSMM is extensively applied to voice recognition [9] and failure prediction [10]. Therefore, HSMM is suitable for modeling and prediction of HSC system.

It is proposed in [5] and [11] that there are two methods of predicting abnormal behaviors of the operator: the observed behavior pattern is similar to a given abnormal behavior pattern; the observed behavior pattern is similar to the normal behavior pattern. In [5] and [12], HMM and HSMM methods are used to conduct modeling for the operator's future behavior and behavior duration; meanwhile, assessment method for this model is established. [5] and [12] both mention that if the operator's behavior deviates from the predicted normal behavior, anomaly might happen. However, neither of them offers the specific method of monitoring the anomaly.

The change of operator's behavior characteristic mode is called "anomaly" here. This paper aims to determine the relation between anomaly and parameters in the model through modeling and thus realize monitoring for abnormal behaviors. If real-time monitoring can be conducted, we will be able to remind the operator and reduce mistakes caused by anomaly of the operator's behavior characteristics.

\section{Methodology}

\subsection{Experimental design}

In order to gain effective experimental data, modeling was carried out for the behavior characteristics. Secondary development was conducted on the basis of RESCHU platform system of MIT, and it was used as experiment platform of our research. Compared with the original platform, UAV framework of the original system was irrelevant to our research, so the UAV type was unified into Medium Altitude Long Endurance (MALE); in addition, relevant parameters were adjusted by directing at the experimental features and record about action distance of each UAV was added. 
30 subjects were selected. After uniform training, the subjects were able to be skilled at RESCHU simulation platform; operational data of each operator was recorded in the database through network. The experimental period lasted for 600s. In order to provide convenience for study on anomaly of the operator's behavior characteristics, the system parameter was changed after 300s, and number of UAVs was changed from 3 to 5.

\subsection{Modeling for the operator's behavior characteristics}

\subsubsection{Definition of observed value}

Records about the experiment platform were summarized, and 26 operations gained in the experiment were generalized into 6 observed values: PAYLOAD_OPERATION (camera operation), GOAL_OPERATION (target operation), WP_OPERATION (path changing operation), VEHICLE_OPERATION (UAV selection operation), BUSY (busy) and IDEL (idle). Observed value could be found for each specific operation behavior, in which BUSY (busy) and IDEL (idle) were used for interpolation.

\subsubsection{Generation of HSMM learning sequence}

Under the scene pressed for time like HSC of UAV, the operation efficiency is very important. An effective learning algorithm proposed by Shun [13] was used here, and this algorithm has small time complexity.

Observed value sequence in this algorithm must be discrete series with equal time interval, and adjacent input observed values represent the same time interval which is the superfine time granularity. Abstract symbol of the observed value was used to generate learning sequence. In this modeling, total length of learning sequence was 600 points $* O_{1} O_{2} O_{3} \ldots O_{600}+$, and the position of each observation point in the sequence represented relative time when this observed value appeared; the value of each observation point was the abstract symbol of observed value (from 1 to 6). Under the superfine granularity, observed values at these time points constituted learning sequence.

\subsubsection{Dynamic learning of HSMM}

Suppose that the time when the operator starts reconnaissance simulation with UAV is 0 . Observed value sequence was generated dynamically till the simulation experiment ended by setting $1 \mathrm{~s}$ as an interval in the future 1s-600s according to the definition and measurement method of observed value introduced above. This process is shown in Table 1:

\begin{tabular}{|c|c|c|c|}
\hline Moment & $\begin{array}{c}\text { Operation } \\
\text { behavior }\end{array}$ & $\begin{array}{c}\text { Observed } \\
\text { value }\end{array}$ & $\begin{array}{l}\text { Termination position of } \\
\text { earning sequence }\end{array}$ \\
\hline \multicolumn{4}{|l|}{$0_{\mathrm{s}}$} \\
\hline $1 \mathrm{~s}$ & & 6(IDEL) & 1 \\
\hline $2 \mathrm{~s}:$ & & 6(IDEL) & 2 \\
\hline 3s: & & 6(IDEL) & 3 \\
\hline 4s: & Goal change & $\begin{array}{l}\text { 2(GOAL_OPER } \\
\text { ATION) }\end{array}$ & 4 \\
\hline 5s: & & 5(BUSY) & 5 \\
\hline 6s: & Goal change end & $\begin{array}{c}\text { 2(GOAL_OPER } \\
\text { ATION) }\end{array}$ & 6 \\
\hline $7 \mathrm{~s}:$ & & 6(DEL) & 7 \\
\hline$\ldots$ & $\ldots$ & $\ldots$ & \\
\hline
\end{tabular}

Table 1: Generation process of learning sequence

From the begging to the end of simulation, the length of observed value sequence would increase from 1 to 600. The number of dynamic learning was 600 (every 1 second) and the learning sequence required by parameter learning was the observed value sequence that was generated. For instance, if parameter learning reaches 500s, the length of learning sequence is the observed value sequence that has been generated in the previous 500s, and the length is 500s.

\subsubsection{Dynamic prediction of HSMM}

After the model parameter $\lambda=\{\pi \mathrm{AB} P\}$ was gained through learning, prediction can be conducted according to the following rule: 
i) At $t$, if the current state $S_{i}$ is known, the observed value $o_{\mathrm{j}}$ at $t$ can be output according to the output probability distribution $b_{i j}$ of the state $S_{i}$;

ii) At $t$, if the current state $S_{i}$ is known and the state of the next moment changes, then the probability will be changed to obey $a_{i j}, j=1,2,3 \ldots M$, where $M$ is number of states and $i$ is the subscript of the current state. Duration of the next state obeys $p_{j k}, k=1,2,3 \ldots D$, where $D$ is the maximum duration of the state and $j$ is the next possible state by following $i$.

Literature [12] defines two dimensions predicted on RESCHU platform by utilizing HSMM: Timing that describes duration of the current observed value; Quality that describes the next observed value which is different. Here this definition was used. However, different from [12], we utilized the method in [18] to acquire Timing and Quality.

\subsection{Evaluation mechanism for dynamic prediction results of HSMM}

Literature [14] proposes a prediction accuracy scoring method for HSMM, which is called Model Accuracy Score (MAS). Different from [14], we conducted accuracy assessment for Timing and Quality respectively and made a separate study. In this way, anomaly of the system could be detected more easily.

\section{Score of Timing:}

Suppose that the current moment is $t$ and duration of predicting the current observed value $O_{t}$ is $d$; in another word, it will take $t+d-1$ to predict $O_{t}$. After the experiment reached the moment of $t+$ $d^{\prime}-1$, the observed value changes, so the actual duration of observed value is $d^{\prime}$. Therefore, the prediction error of observed value duration at $t$ is $\varepsilon_{t}=\left|d-d^{\prime}\right|$. Suppose that before $t$, duration of $O_{t}$ obeys Gaussian distribution $N\left(x, \mu_{o t}, \varsigma_{o t}\right)$; the mean value $\mu_{o t}$ and standard deviation $\varsigma_{o t}$ of the duration of $O_{t}$ is approximated to mean value and standard deviation of Gaussian distribution. Therefore, the score of Timing $T$ is:

\section{Score of Quality:}

$$
T=200 \times \int_{\mu_{0 t}+\varepsilon_{t}}^{\infty} N\left(x, \mu_{0 t}, \sigma_{0 t}\right) d x
$$

Suppose that the current moment is $t$, and the next observed value of the current observed value $O_{t}$ is $O_{n}\left(O_{\text {next }} \neq O_{t}\right)$. Quality $=\left(p_{5}, p_{3}, p_{2}, p_{4}, p_{1}, p_{6}\right)$, where $P\left(\right.$ next observation $\left.=O_{\text {next }}\right)=$ $p_{\text {onext }}, p_{5} \geq p_{3} \geq p_{2} \geq p_{4} \geq p_{1} \geq p_{6}, p_{o} t=0$, and $\sum_{0 \neq 0 t} p_{0}=1$. Suppose that the next different actual observed value is $O_{\text {realnext }}=2$. The score of Quality is:

$$
Q=100 \times\left(p_{2}+p_{4}+p_{1}+p_{6}\right)
$$

When the actual result is located at the first dimension of Quality, the score $Q$ is 100 . When it is located at a certain dimension in the middle, the score meets the relation between probability accumulation and probability.

$$
Q=100 \times \sum_{j=i}^{6} \text { Quality }(j)
$$

\subsection{Collection of prediction results}

There were 30 experiment samples in the scene of "3 UAVs for 10 minutes". For each experiment sample, dynamic learning was conducted from 2 states to 8 states, and then prediction was carried out. Prediction result of each operation under each state (score of Timing or Quality) was a sequence with a length of 600. Each point of the sequence represents the score of Timing or Quality at the operation moment. The following formula is a better organizational form of displaying the data.

$$
\begin{aligned}
& \mathrm{T}=\left(\begin{array}{ccc}
T_{1,1} & \cdots & T_{1,7} \\
\vdots & & \vdots \\
T_{30,1} & \cdots & T_{30,7}
\end{array}\right)_{30 \times 7} \\
& \mathrm{Q}=\left(\begin{array}{ccc}
Q_{1,1} & \cdots & Q_{1,7} \\
\vdots & & \vdots \\
Q_{30,1} & \cdots & Q_{30,7}
\end{array}\right)_{30 \times 7}
\end{aligned}
$$

$T_{i, j}$ or $Q_{i, j}$ are vectors with the length of 600 . Each point represents the dynamic prediction result of operator $i$ when number of HSMM model states is $j+1$. 


\section{4. analysis and discussion of experimental results}

\subsection{The change rule of prediction effect values of Timing and Quality with the operation time}

The prediction sequence of all operators under all states was averaged and central moving average was conducted via the window with a length of 51 . The following average sequence expressed by Timing and Quality was gained, where

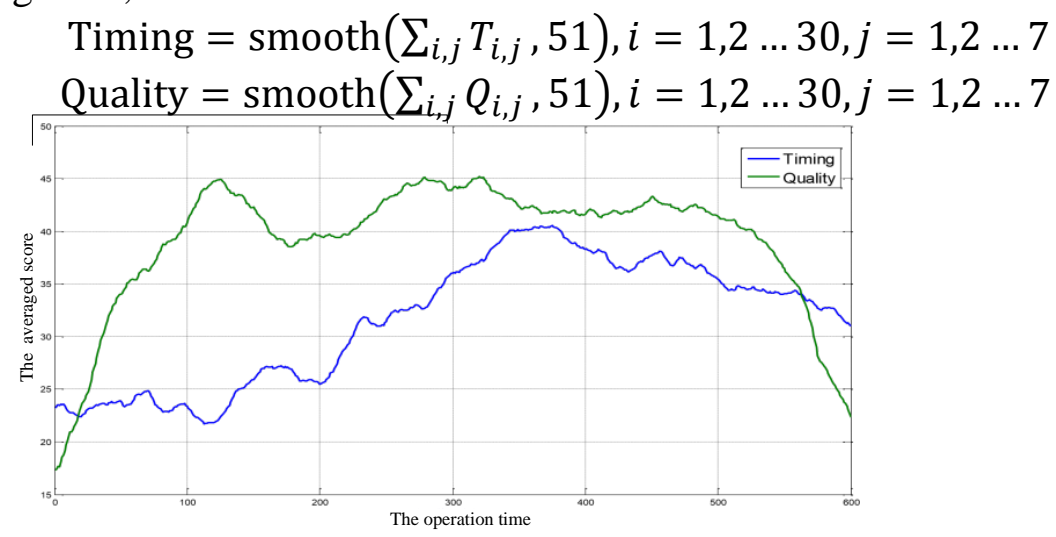

Figure 2: Change of Timing and Quality with time

As shown in Fig. 2, as the operation time passes, length of the learning sequence increases; Timing and Quality rise at first and then decrease. At the initial stage of the experiment, the score of Quality almost presents a trend of linear increase; after reaching the peak value, it maintains smooth and steady. After about 300s, it decreases slowly and finally drops sharply. At the initial stage, Timing rises slowly and decreases slowly after reaching the peak value.

\subsection{Analysis on anomaly of behavior characteristics}

Change of the operator's behavior pattern is called "anomaly”. Due to "anomaly”, HSMM generated by learning sequence of behavior characteristics at the previous stage cannot accurately predict current behaviors of the operator, thus the corresponding score of MAS will be quite low. According to the experimental design, number of UAV starts to change after 300s, which is bound to result in some changes of the operator's behavior characteristics, anomaly in another word.

\subsubsection{Experimental verification}

In order to verify that what reduces the prediction effect of HSMM is anomaly of the operator's behavior characteristics rather than other factors, we made another group of experiments, with the code of $\tilde{B}$. In this group of experiments, platform parameters were the same with the previous experiments during the first 300s; after 300s, the maximum value of learning sequence length was set as 300 and a comparison was made with the previous group of experiments whose code was $\tilde{A}$. The learning sequence increased synchronously with time. Fig. 3 presents the correlation curves about variation of central moving average operation time for the average values of all experimenters' Timing and Quality under all states:

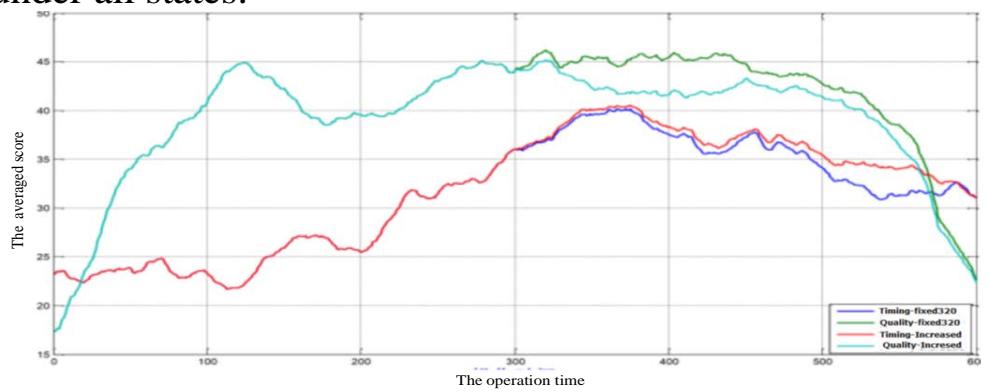

Figure 3: Contrast between $\tilde{A}$ and $\tilde{B}$ curves 


\subsubsection{Determination for anomaly characterization parameters of the operator's behavior characteristics}

According to Fig. 4, before 300s, learning sequence length of group $\tilde{A}$ is always equal to that of group $\tilde{B}$, so the prediction results are the same. After $300 \mathrm{~s}$, obvious anomaly happens to behavior characteristics of all operators when compared with the previous 300s. In group $\tilde{A}$, the learning sequence keeps rising, and its prediction result is inferior to that of group $\tilde{B}$ whose maximum learning sequence length and anomaly duration are the same. Therefore, the best prediction effect can be realized only when the learning sequence length is equal to the time interval after anomaly happens.

By observing the prediction effect of group $\tilde{A}$ at the stage 300s-400s after anomaly appears, obvious fluctuation happens to Quality curve of group $\tilde{A}$ when compared with group $\tilde{B}$, and later it tends to be steady. This shows that the anomaly has resulted in huge change of the operator's behavior patterns, which has reduced the model prediction effect.

When learning sequence length contains excessive "past” information, Quality prediction accuracy of the corresponding learning sequence reduces while the prediction accuracy of Timing increases. Thereby, Quality is sensitive to "current" information and it can effectively show the change of the operator's current behavior characteristics, which is anomaly. However, Timing is only sensitive to "past" information, and the operator's characteristic of "current observation duration" does not change greatly with time; therefore, it cannot show the anomaly.

We verified that Quality was sensitive to the current anomalous change by taking operator 26 as an example. Fig. 4 depicts the difference between $\tilde{A}$ matrix and $\tilde{B}$ matrix whose learning sequences are 1s-300s and 300s-600s respectively. According to the observation, even the basic form of the matrix changes and the difference is obvious. Thereby, $\tilde{A}$ matrix and $\tilde{B}$ matrix that reflect the operator's behavior characteristics have a great difference between the first half stage and the second half stage. Therefore, obvious differences happen to the prediction results of Quality.

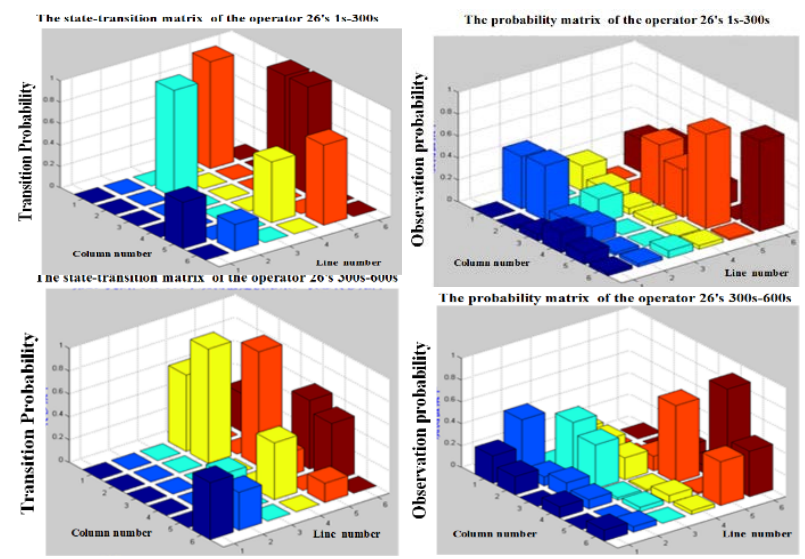

Figure 4: The difference of $\tilde{A}$ matrix and $\tilde{B}$ matrix in the first 300s and last 300s

\subsection{Monitoring methods for the operator's abnormal behavior}

In the previous section, we pointed out that Quality could conduct real-time characterization for the change of behavior pattern which was "anomaly". In practical monitoring, similar to group $\tilde{A}$ in the control experiment, there is a great uncertainty due to anomaly and the learning sequence can increase only gradually. When anomaly appears, the operator's behavior characteristics will change and the originally stable Quality prediction evaluation curve will decrease obviously. Evaluation for anomaly of the operator's behavior characteristics can be realized by observing Quality.

\section{Conclusions}

This paper established an improved HSMM prediction model and prediction accuracy assessment method on the basis of the original researches under the drive of experimental data on the RESCHU simulation platform, and this model is more suitable for anomaly detection of the operator's behavior characteristics. By designing the contrast experiment, the relation between abnormal behavior and 
model prediction was studied. Meanwhile, it verified that anomaly of behavior characteristics was the major factor of changing the prediction effect of HSMM, and Quality could be used as the major evaluation parameter to monitor anomaly of the operator's behavior characteristics. Besides, we also determined the method of monitoring anomaly, and anomaly of the current operation could be effectively reflected by observing the change of Quality. This model method can be used to monitor anomaly of the operator's behavior characteristics in practical HSC system. In actual system, it is able to enhance the operator's efficiency and reduce human errors.

\section{References}

[1] Dixon, S.R., C.D. Wickens (2003). Control of Multiple-UAVs: A Workload Analysis.

[2] Rodas M O, Szatkowski C X, Veronda M C. Predicting an adequate ratio of unmanned vehicles per operator using a system with a mission definition language[C]//Cognitive Methods in Situation Awareness and Decision Support (CogSIMA), 2011 IEEE First International MultiDisciplinary Conference on. IEEE, 2011: 159-162.

[3] Sheridan, T.B., 1992. Telerobotics, Automation and Human Supervisory Control.The MIT Press, Cambridge, MA.

[4] http://web.mit.edu/aeroastro/labs/halab/inventions.shtml\#RESCHU

[5] Boussemart Y, Cummings $\mathrm{M}$ L. Behavioral recognition and prediction of an operator supervising multiple heterogeneous unmanned vehicles[J]. Humans operating unmanned systems, 2008.

[6] G. Klein, Sources of Power: How People Make Decisions. Cambridge, MA:The MIT Press, 1999.

[7] Welford, A.T., 1952. The psychological refractory period and the timing of highspeed performance-a review and a theory. British Journal of Psychology 43,2-19.

[8] Rabiner, L.R., 1989. A tutorial on Hidden Markov Models and selected application in speech recognition. Proceedings of the IEEE 77 (2), 257-286.

[9] Takahashi Y, Tamamori A, Nankaku Y, et al. Face recognition based on separable lattice 2-D HMM with state duration modeling[C]//Acoustics Speech and Signal Processing (ICASSP), 2010 IEEE International Conference on. IEEE, 2010: 2162-2165.

[10] Dong M, He D. Hidden semi-Markov model-based methodology for multi-sensor equipment health diagnosis and prognosis[J]. European Journal of Operational Research, 2007, 178(3): 858-878.

[11] Singh, S., Tu, H., Donat, W., Pattipati,, K., Willet, P., 1996. Anomaly detection via featureaided trạcking and hidden Markov models. IEEE Transactions on Systems, Man, and Cybernetics.

[12] Yves Boussemart 2010 Predictive models of human supervisory control behavioral patterns using hidden semi-Markov models

[13] Shun-ZhengYu and Hisashi Kobayashi , Life Fellow, IEEE 2006 Practical Implementation of an Efficient Forward-Backward Algorithm for an Explicit-Duration Hidden MarkovModel

[14] Hank Hsin Han Huang 2009 Developing an Abstraction Layer for the Visualization of HSMMBased Predictive Decision Support 